\title{
Nurses' Clinical Judgment Development: A Qualitative Research in Iran
}

\author{
Jamal Seidi, ${ }^{1,2}$ Fatemeh Alhani, ${ }^{1, *}$ and Mahvash Salsali ${ }^{3}$ \\ ${ }^{1}$ Department of Nursing, Faculty of Medical Sciences, Tarbiat Modares University, Tehran, IR Iran \\ Department of Nursing, Faculty of Medical Sciences, Tarbiat
${ }_{2}$ Kurdistan University of Medical Sciences, Sanandaj, IR Iran \\ ${ }_{3}^{3}$ Kepartment of Medical and Surgical Nursing, School of Nursing and Midwifery, Tehran University of Medical Sciences, Tehran, IR Iran \\ ${ }^{*}$ Corresponding Author: Fatemeh Alhani, Department of Nursing, Faculty of Medical Sciences, Tarbiat Modares University, Tehran, IR Iran. Tel: +98-2182883898, Fax: +98-2182883856, \\ E-mail: alhani_f@modares.ac.ir
}

Received: May 23, 2014; Revised: June 21, 2014; Accepted: July 3, 2014

Background: Clinical judgment development is necessary because it leads to appropriate nursing diagnoses, clinical decision-making and health promotion.

Objectives: In this study we explored the process of Iranian nurses' development in clinical judgment.

Patients and Methods: This qualitative study was conducted in 2013 at hospitals of Kurdistan University of Medical Sciences, located in the Sanandaj city of Iran. The data were collected based on semi-structured interviews and the study included 24 participants. Data analysis was carried out concurrently with data collection using the grounded theory method.

Results: The study participants' main concern was 'being non-professional in clinical judgment'. In response to this concern, they were struggling for gaining professional autonomy, striving for integrating clinical judgment skills, scrambling to make effective educational interventions and striving for professional and inter professional collaboration in clinical judgment. The core category was 'struggling for becoming professional in clinical judgment development'. When nurses were supported professionally, they were able to develop their professional clinical judgment.

Conclusions: The findings of this study provided critical information about nurses' professionalization in clinical judgment. Accordingly, the participants adopted different strategies to develop their clinical judgment ability. Integrating these strategies into nursing theory and clinical education can improve nurses' clinical judgment ability.

Keywords: Judgment; Nurse; Qualitative Research; Iran

\section{Background}

Clinical judgment is one of the key attributes of professional practice (1). It is a prerequisite for establishing professional identity (2) and is mainly based on nurses' knowledge and experience as well as their reasoning, intuition, clinical thinking, and evidence-based practice skills (3, 4). Nurses use these skills to assess patients and the environment and to process and interpret patient information in order identify and fulfill patient needs (5). This process results in the establishment of nursing diagnoses, effective clinical decision-making, problem solving and the improvement of care quality (6). Tanner (5) noted that only professional nurses could go through this process. Such a structured approach to patient care is an important characteristic of structured professional judgment (7). Professionalization (8) in clinical judgment is a major challenge of modern nursing (7) and despite its critical importance, it is still poorly understood.

Previous studies have explored and identified some of the main characteristics of nurses' professional practice, clinical judgment, and clinical decision-making. These characteristics included using knowledge and conceptual models in practice $(9,10)$, adopting an interdisciplin- ary collaborative decision-making approach (9), including clinical judgment in nursing educational programs $(11,12)$, and using evidence, intuition and autonomy (13). Gerdeman et al. (11) noted that nurses' skillfulness is one of the main characteristics of professional nursing practice. Nurses' skillfulness pertains to their ability to use their skills in different situations. In other words, nurses' ability to use knowledge, experience and evidence, as well as their critical thinking skills in daily practice reflects their skillfulness in clinical judgment.

Autonomy is another characteristic of professional nursing practice (13). Professional autonomy is nurses' ability to establish relationships and interact with others and to use knowledge and wisdom in daily clinical practice (14). Gillet et al. (15) conducted a survey study on 500 French nurses and reported that procedural justice and supervisors' autonomy support, positively affects nurses' satisfaction and perceived organizational support.

Implementing educational programs for improving nurses' clinical judgment is another characteristic of professional nursing practice. Kantar and Alexander (16) inte-

Copyright ( 2015, Iranian Red Crescent Medical Journal. This is an open-access article distributed under the terms of the Creative Commons Attribution-NonCommercial 4.0 International License (http://creativecommons.org/licenses/by-nc/4.0/) which permits copy and redistribute the material just in noncommercial usages, provided the original work is properly cited. 
grated clinical judgment into nursing curriculum and reported that education enhances nurses' clinical judgment skills (17). Other educational interventions such as conceptualizing (18) and simulation (19) as well as teaching critical thinking skills (20) can also improve nurses' clinical judgment ability.

Another characteristic of professional nursing practice is interdisciplinary and interdisciplinary collaboration. Lockwood et al. (21) reported that nurses' collaboration with other healthcare providers plays an important role in their professionalization. Moreover, interdisciplinary collaboration between clinical nurses and nurse educators can improve nurses' clinical judgment skills $(17,22)$.

Despite the importance of clinical judgment in nursing, our critical appraisal of the literature revealed that previous studies have examined only specific aspects of the concept or compared certain types of this concept, mainly by using quantitative approaches in nursing. Moreover, there are very few qualitative studies on clinical judgment development in nursing. The conducted qualitative studies have also focused mainly on isolated areas of clinical judgment. For example, Elliott (23) conducted a grounded theory study to explore the issues of nurse-patient interaction in clinical judgment. Accordingly, the process of nurses' clinical judgment development is hardly known. We conducted the current study to bridge this knowledge gap.

\section{Objectives}

The aim of this study was to explore the process of Iranian nurses' development in clinical judgment.

\section{Patients and Methods}

In this qualitative study we used a grounded theory approach, which is the best method for exploring complex social processes and individuals' main and common concerns (23). Moreover, clinical judgment is an interactive process and such processes are the core component of the symbolic interaction theory (23). This study was conducted during years 2013 and 2014.

\subsection{Data Collection}

We collected the study data by using the semi-structured interview method. The main interview questions included but were not limited to the following;

a) Would you please explain about your patient assessment practice during a workday?

b) What types of experiences do you remember when you hear 'clinical judgment'?

c) Which factors and behaviors help improve nurses' clinical judgment?

d) How do you help improve your own and your colleagues' clinical judgment?

We also used probing questions to broaden our understanding about participants' experiences. Moreover, we complemented the study data by attending the clinical settings and observing nurses' clinical judgment practice. The researcher who was the interviewer was familiar with the method of the study and he interviewed the participants faceto-face. The findings of each interview and the recorded observations guided the subsequent interview or observation. We continued the data collection until data saturation, i.e. until development of all the emerged concepts and categories and discovery of the relationships between them. We scheduled the interviews according to the participants' preferences and convenience. To clarify ambiguities in the conducted interviews, we interviewed five participants twice. Consequently, 29 interviews were performed. Interviews lasted 30 - 60 minutes.

\subsection{Setting and Participants}

Nurses with at least three years of work experience in clinical nursing who were willing to participate in the study and share their experiences were considered as key informants. The sampling method was initially purposive. Thereafter, we employed the theoretical sampling method to develop the emerging concepts and categories. We employed maximum variation sampling method to select different Participants (Table 1) working in different clinical settings of Kurdistan University of Medical Sciences located in the Sanandaj city of Iran.

\begin{tabular}{|c|c|}
\hline Variable & Values \\
\hline \multicolumn{2}{|l|}{ Gender } \\
\hline Female & $12(50)$ \\
\hline Male & $12(50)$ \\
\hline \multicolumn{2}{|l|}{ Level of education } \\
\hline BSc & $13(54.2)$ \\
\hline MSc & $3(12.5)$ \\
\hline PhD & $2(8.3)$ \\
\hline Doctorate & $1(4.2)$ \\
\hline Pediatrics & $1(4.2)$ \\
\hline Diploma & $2(8.3)$ \\
\hline Fourth-year student & $2(8.3)$ \\
\hline \multicolumn{2}{|l|}{ Occupation } \\
\hline Clinical nurse & $14(58.4)$ \\
\hline Head nurse & $1(4.2)$ \\
\hline Educator nurse & $2(8.3)$ \\
\hline Supervisor & $1(4.2)$ \\
\hline Physician & $2(8.3)$ \\
\hline Nursing student & $2(8.3)$ \\
\hline In-patient and family member & $2(8.3)$ \\
\hline \multicolumn{2}{|l|}{ Work experience, y } \\
\hline $3-10$ & $7(29.2)$ \\
\hline $11-20$ & $13(54.2)$ \\
\hline$>20$ & $4(16.6)$ \\
\hline \multicolumn{2}{|l|}{ Age, $y$} \\
\hline $23-33$ & $4(16.6)$ \\
\hline $34-45$ & $14(58.4)$ \\
\hline$>45$ & $6(25)$ \\
\hline
\end{tabular}


Inclusion criteria included willingness to participate in the study and having full experience in clinical judgment. Exclusion criteria included withdrawal from the study at any time for any reason. We included 25 participants in the study. One nurse who was the mother of a seriously ill child refused to participate, which ended in her exclusion from the study. In general, 24 participants took part in the study (Table 2).

\subsection{Ethical Considerations}

The ethics committee of Tarbiat Modares university, Tehran, Iran, approved the study (No: 52/112071). The study participants were clearly informed about the aims of the study. We guaranteed the confidentiality of the participants' personal information. The study partici- pants were completely free to participate or withdraw from the study. Finally, we obtained a written informed consent from each participant.

\subsection{Data Analysis}

Constant comparative analysis of old and new data and use of analytic tools helped us minimize the effects of biases on the analysis process. The employed analytic tools included questioning, constant comparisons, using the flip-flop technique, thinking about different meanings of words, drawing upon personal experiences, waving the red flag, and looking at language. This approach to analysis increased our theoretical sensitivity during data collection and analysis. We managed the study data using the MAXQDA10.0 software.

\begin{tabular}{|c|c|c|c|c|c|c|}
\hline $\begin{array}{l}\text { Participants' } \\
\text { Codes }\end{array}$ & Occupation & Gender & Age & Level of Education & Work Experience, $y$ & Setting \\
\hline P1 & Clinical nurse & Female & 36 & BSc & 16 & Psychiatric ward \\
\hline P2 & Clinical nurse & Male & 33 & MSc & 7 & ICU \\
\hline P3 & Head nurse & Male & 33 & BSc & 21 & ICU \\
\hline P4 & Educator nurse & Male & 32 & $\mathrm{PhD}$ & 9 & $\mathrm{CCU}$ \\
\hline P5 & Clinical nurse & Male & 28 & BSc & 8 & Operating room \\
\hline P6 & Clinical nurse & Male & 35 & BSc & 11 & Neurosurgery ward \\
\hline P7 & Clinical nurse & Male & 34 & BSc & 14 & Psychiatric ward \\
\hline P8 & Supervisor nurse & Male & 44 & BSc & 20 & Nursing office \\
\hline P9 & Educator nurse & Female & 42 & $\mathrm{PhD}$ & 17 & Nursing colleague \\
\hline P10 & Clinical nurse & Female & 26 & MSc & 3 & $\mathrm{CCU}$ \\
\hline P11 & Clinical nurse & Male & 38 & BSc & 16 & Surgical ward \\
\hline P12 & Clinical nurse & Female & 35 & BSc & 11 & Neurosurgery ward \\
\hline P13 & Clinical nurse & Female & 40 & BSc & 20 & Emergency ward \\
\hline P14 & Clinical nurse & Female & 42 & BSc & 22 & Post partum ward \\
\hline P15 & Clinical nurse & Female & 38 & BSc & 16 & Medical ward \\
\hline P16 & Clinical nurse & Female & 28 & MSc & 6 & ICU \\
\hline P17 & Clinical nurse & Female & 38 & BSc & 16 & Surgical ward \\
\hline P18 & Clinical nurse & Female & 39 & BSc & 16 & Neurosurgery ward \\
\hline P19 & Physician & Male & 45 & Doctorate & 18 & Emergency ward \\
\hline P20 & Pediatrics & Male & 50 & Doctorate & 20 & Neonatal ICU \\
\hline P21 & Nursing student & Female & 24 & Fourth-Year Student & 3 & Emergency ward \\
\hline P22 & Nursing student & Male & 26 & Fourth-Year Student & 3 & Operating room \\
\hline P23 & In-patient & Male & 32 & Diploma & $\mathrm{a}$ & Emergency ward \\
\hline P24 & Family Member & Female & 30 & Diploma & $\mathrm{a}$ & Pediatric ward \\
\hline
\end{tabular}

\footnotetext{
${ }^{a}$ Having sufficient experience of hospitalization.
} 


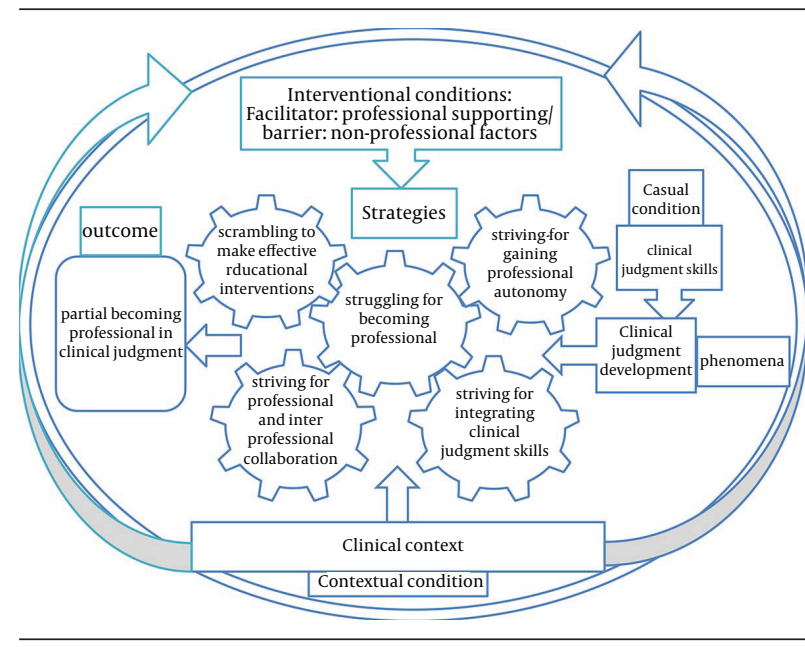

Figure 1. Nurses' Clinical Judgment Development Model

\subsection{Consideration of Rigor}

To establish the credibility of the study findings, we performed data analysis concurrently with data collection and employed the constant comparison analysis, active listening, prolonged engagement and immersion techniques, as well as member-checking. Moreover, we documented and kept a record regarding all of our analytic activities for the purpose of dependability. To ensure de- pendability we employed the triangulation technique. In triangulation, we collected the study data by using both the interview and the observation methods. To ensure the confirmability of the study findings, we employed the peer-check method. Finally, we recruited a maximum variation sample to improve the transferability of the study findings.

\section{Results}

The study participants' main concern was 'being nonprofessional' in clinical judgment development. In response to this concern, they were 'struggling for becoming professional'. This was the study's core category encompassing and linking all the other categories. The consequence of this process was partial professionalization in clinical judgment development. When nurses were supported professionally, they were able to develop their professional clinical judgment. However, in case of growing presence of non professional factors, they were unable to openly practice their clinical judgment skills (Figure 1). The properties or sub-categories of the study core category were 'striving for gaining professional autonomy', 'striving for integrating clinical judgment skills', 'scrambling to make effective educational interventions', and 'striving for professional and inter professional collaboration' (Table 3). These categories are explained below.

Table 3. The Core, Categories and Sub-Categories Under Study ${ }^{\text {a }}$

Categories $^{b}$ Primary Codes

Striving for gaining professional autonomy

1-Making independent nursing interventions

2-Creating a distinctive professional identity

3-Gaining nurses' proficiency in using their own skills

4-Creating a culture for independent presence of nurses in clinical judgment

Striving for integrating clinical judgment skills

1-Integrating clinical judgment skills based on patients' condition and the immediate situation

2-Integrating clinical judgment skills based on kinds of evidences

\section{Scrambling to make effective educational interventions}

1-Integrating clinical judgment into nursing curriculum and clinical education

$4(44)$

2-Integrating nursing and medical education in terms of clinical judgment

\section{Striving for professional and inter professional collaboration}

1-Interpersonal collaboration in clinical judgment activities

2-Interdisciplinary collaboration in clinical judgment activities

3-Involving patients and their family members in clinical judgment

$2(10)$

a Data are presented as No. (\%).

b Core category is struggling for becoming professional in clinical judgment. 
Seidi J et al.

\subsection{Striving for Gaining Professional Autonomy}

Our participating nurses were willing to make independent nursing interventions, practice an independent profession, and create a distinctive professional identity. They preferred autonomy over dependence and inferiority. Consequently, they strived to adopt different strategies to gain autonomy. One of their strategies was to develop a specialized curriculum both for the theoretical and clinical nursing education. They believed that such a curriculum could help them make independent clinical judgments and hence, gain autonomy. A nurse said, "after completing specialized trainings in intensive care, I acquired better clinical judgment skills and now I can make more accurate clinical judgments".

Nurses also noted that gaining more autonomy from medicine, in terms of nursing interventions, would help them make independent clinical judgments. Accordingly, they had adopted strategies such as providing patient care based on nursing models and theories as well as clinical practice guidelines to enhance their autonomy. A practicing nurse said, "Providing care to patients based on Orem's model helped us make autonomy independently from physicians". Another nurse added, "One of our patients was experiencing urinary retention. We, based on clinical practice guidelines, could judge whether the patient needed suprapubic bladder massage or any other intervention".

Nurses' proficiency in using their own clinical knowledge, experience, and critical thinking skills as well as clinical evidence also helped them make independent clinical judgments. A practicing nurse said, "My reasoning about patient's condition was based on my clinical knowledge and experience. Attending physicians also respected and referred to my judgments". Our participants were also striving for creating a culture for independent presence of nurses in clinical judgment. In an attending physician's words, "In my TV talks, I tried to present nurses' autonomy in clinical judgment".

\subsection{Striving for Integrating Clinical Judgment Skills}

Our participants' next strategy for becoming professional was striving for integrating clinical judgment skills. The participating nurses strived to use different clinical judgment skills based on patients' condition and the immediate situation. For example, in emergency situations, if there was a lack of clinical guidelines, experienced nurses integrated their knowledge and experience for making accurate clinical judgments. A practicing nurse said, "Immediately after delivery, the mother developed pulmonary emboli. As we had foreseen this problem based on our previous experiences, we immediately performed cardiopulmonary resuscitation and she revived".

In emergency situations, inexperienced nurses had no option but to rely on physicians' knowledge and experi- ence to make clinical judgment. A practicing nurse affirmed this reliance on physicians when she said, "At the emergency department, we had no guideline for making clinical judgment about patients needing Computerized Tomography (CT) scan. Consequently, we were compelled to make judgment based on the attending physician's experience". In emergency situations, if nurses had access to guidelines, they made clinical judgments by integrating guidelines and their own knowledge and experience. A practicing nurse said," Based on Cardiopulmonary Resuscitation (CPR) guidelines, our judgment was that the patient needed cardio version. Our previous experience also confirmed this".

In normal conditions, however, nurses' approach to integrating their clinical judgment skills was different from emergency situations. In such conditions, nurses integrated their own knowledge and experience, clinical guidelines, and recent research findings for making judgment. Moreover, they also referred to nursing textbooks. At the ward of chronic diseases care, a nurse said, "For applying dressing on a bed sore, I referred to the findings of a research paper. My colleague shared his experiences and the attending physician provided his viewpoint. Finally, we reached a final agreement on the dressing technique".

\subsection{Scrambling to Make Effective Educational In- terventions}

Our participating nurse educators were scrambling to integrate clinical judgment into nursing curriculum and clinical education. A nurse educator claimed, "I am trying to teach this concept to my students during both theoretical and clinical educational courses". Nurse Managers and practicing nurses, on the other hand, were also scrambling to include the concept of clinical judgment in continuing education, patient education, and self-learning programs. A head nurse added, "We are scrambling to develop and offer courses for improving nurses' clinical judgment skills". Another strategy adopted by our participants was to integrate nursing and medical education in terms of clinical judgment. Another nurse educator said, “To improve nurses' clinical judgment skills, I tried to schedule and hold joint conferences among practicing nurses, nursing students, and attending physicians".

\subsection{Striving for Professional and Inter Professional Collaboration}

Our participating nurses, nursing students, nurse educators, physicians, and patients and their family members were striving for interpersonal and interdisciplinary collaboration in clinical judgment activities. Physicians' willingness to collaborate with nurses mainly depended on nurses' clinical judgment ability. Physicians had great confidence in experienced nurses' skills. A practicing nurse said, "When doing patient assessment, the attending 
physician paid attention to my nursing report and the patient's history that I had recorded. We interacted with each other".

Nurses' participation in activities greatly depended on their self-confidence and clinical judgment ability. Experienced and confident nurses actively collaborated with physicians in making accurate clinical judgments. A practicing nurse mentioned, "When interacting with the attending physician, I tried to use reasoning and to refer to clinical evidence for sharing my opinion". Another said, "Some of our nurses also strive to involve patients and their family members in clinical judgment". Another practicing nurse said," At the psychiatric ward, we record the patient history by questioning both the patients and their family members. We sought their opinions".

Depending on their awareness of patient's condition, patients and their family members were sometimes willing to participate in the clinical judgment process. A family member affirmed his collaboration when he said, "I told the nurse, 'my father has allergy to Salbutamol'. She informed the doctor and the doctor prescribed an alternative medication".

Nurse educators were also striving to foster others' collaboration. They usually involved other parties in their clinical judgment education. A shift supervisor said, "To improve nurses' clinical judgment skills, we held joint morning report sessions. We tried to involve physicians, nurse educators, and nursing students in these sessions".

\section{Discussion}

In this study, we aimed to explore the process of Iranian nurses' professionalization in clinical judgment. The study findings revealed that the participants employed different strategies for becoming professional in clinical judgment development. These strategies constituted the core category of the study, which was 'struggling for becoming professional in clinical judgment'. To the best of our knowledge, none of the previous studies had explored the process of professionalization in clinical judgment. However, several studies have reported some of the properties and strategies of professionalization in clinical judgment $(12,24)$.

We found that one of the strategies employed by our participants for becoming professional in clinical judgment was striving for gaining professional autonomy. The participating nurses were striving for specialization in nursing and for making independent nursing interventions. These strategies helped nurses gain autonomy in clinical judgment. Petrucci et al. (25) reported a significant correlation between residents' skillfulness and their ability to make accurate clinical judgments. Our participating nurses tried to provide patient care based on nursing models and theories and clinical practice guidelines. Gampel (26) reported that independent interventions can result in accurate clinical judgment only when they are designed and implemented based on pro- fessional standards. The study findings also revealed that nurses used clinical evidence as well as their own clinical knowledge, experience, and critical thinking skills to gain autonomy in clinical judgments. Traynor et al. (24) noted that evidence-based practice and experiencebased intuition improve nurses' autonomy in clinical judgment. They also found that nurses struggled with organizational authorities for maintaining their professional autonomy (24). Our participants were also striving for developing a culture for improving nurses' autonomy in clinical judgment. Wang et al. (27) found that sociocultural factors interfered with nurses' professional autonomy in clinical judgment.

We found that another strategy used by nurses for becoming professional was the integration of different clinical judgment skills in different situations. In case of limited access to clinical guidelines, more experienced nurses were able to use their intuition and critical thinking skills to make accurate clinical judgments and handle the situation. Dickson (28) described how the nurses integrated different knowledge and skills to make decisions in the medication administration process. Clarke (29) found that surgical residents who were able to use their clinical skills according to patient's conditions made clinical judgments that were more accurate. Cranley et al. (30) also found that nurses with higher ability in integrating different skills were more able to recognize and manage uncertainties in their practice. In a study aimed at evaluating implicit clinical judgment, Nakash and Alegria (31) found that clinical judgment based on experience and knowledge could lead to the integration of information.

Our participating nurses were also scrambling to make effective educational interventions (such as including the concept of clinical judgment in the nursing curriculum as well as continuing education, patient education, and self-learning programs) to develop their clinical judgment ability. Lasater (32) noted that the patterns of clinical judgment need to be integrated in students' professional conduct. In previous studies, different educational interventions including clinical simulation (33), concept mapping (11), and continuing education programs (34) were developed and implemented for promoting clinical judgment. Nielsen (35) reported that concept-based learning activities designed based on the Tanner clinical judgment model enhanced students' clinical judgment skills and helped them move form novice to experts. Lasater (32) reported that to maximize the effectiveness of simulation in developing students' clinical judgment skills, simulation-based educational programs need to be integrated into clinical education and practice. Lasater and Nielsen (36), Banning(37)also noted that incorporating experienced nurses and nurse educators into clinical judgment educational interventions helps expand students' understanding of clinical judgment.

Striving for professional and inter professional collaboration was another strategy adopted by our participants for clinical judgment development. The collaboration was 
among all parties involved in patient care including physicians, nurses, patients, and their family members. Parker et al. (38) noted that collaboration between Nurses and other staff could lead to avoid errors. According to Legare et al. (9), interdisciplinary collaboration and interpersonal interaction in the process of clinical decision-making and clinical judgment are the key attributes of nurses' professionalism. Interdisciplinary collaboration among nurses, specialists, nurse educators, and nursing students is an absolute prerequisite for developing critical thinking and clinical judgment skills $(39,40)$. Elliot (23) also noted that for making accurate clinical judgments, nurses need to closely collaborate with patients and their families. Collaboration between nurses and patients and their families result in higher level of satisfaction (41).

Finally, the study findings revealed that participants employed different strategies for clinical judgment development. These strategies constituted the core category of the study, which was 'struggling for becoming professional. To the best of our knowledge, none of the previous studies explored the process of professionalization in clinical judgment. However, several studies have reported on some of the properties and strategies of professionalization in clinical judgment $(12,13)$. Further studies are needed to fully understand the process of professionalization in clinical judgment development. Investigating the effectiveness of integrating clinical judgment into nursing curriculum and continuing education programs is also recommended.

Results of this qualitative research have limited transferability. We strived to manage our experiences and viewpoints by 'drawing on personal experiences'; however, these may affect our approach towards data collection methods and appropriate analysis. On the other hand, the sincere cooperation of the participants was a strong point of this study.

\section{Acknowledgements}

The authors thank all the participants who agreed to participate in this study. This study was written based on the first investigator's PhD dissertation; therefore, the financial support from the faculty of Medical Sciences, Tarbiat Modares university is also acknowledged.

\section{Authors' Contributions}

Jamal Seidi was the main investigator and contributed to the development of the study concept and design, acquisition of data, analysis and interpretation of data and drafting of the manuscript. Fatemeh Alhani supervised the study and contributed to the development of data collection, analysis and interpretation of data and revision of the manuscript. She also provided administrative, technical, and material support for this study. Mahvash salsali was the advisor of the study, contributed to the analysis and interpretation of data and prepared and revised the manuscript.

\section{Funding/Support}

This study was written based on the first investigator's PhD dissertation, which had received a grant(No: 52/112071) from the department of nursing, faculty of medical sciences, Tarbiat Modares university, Tehran, Iran.

\section{References}

1. Kelly MA, Hager P, Gallagher R. What matters most? Students' rankings of simulation components that contribute to clinical judgment. J Nurs Educ. 2014;53(2):97-101.

2. Glick M. Clinical judgment: a requirement for professional identity. J Am Dent Assoc. 2011;142(12):1333-4.

3. Benner P, Tanner CA, CHesla CA. Expertise in Nursing Practice Caring, Clinical Judgment \& Ethics. New York, NY10036-8002: Springer Publishing Company, LLC;2009. p. 47.

4. Tanner CA. Clinical judgment and evidence-based practice: toward pedagogies of integration. J Nurs Educ. 2008;47(8):335-6.

5. Tanner CA. Thinking like a nurse: a research-based model of clinical judgment in nursing. J Nurs Educ. 2006;45(6):204-11.

6. Pongmarutai T. Application of a judgment model toward measurement of clinical judgment in senior nursing students. Las Vegas: University of Nevada; 2010.

7. Falzer PR. Valuing structured professional judgment: predictive validity, decision-making, and the clinical-actuarial conflict. Behav Sci Law. 2013;31(1):40-54.

8. ten Hoeve Y, Jansen G, Roodbol P. The nursing profession: public image, self-concept and professional identity. A discussion paper. J Adv Nurs. 2014;70(2):295-309.

9. Legare F, Stacey D, Gagnon S, Dunn S, Pluye P, Frosch D, et al. Validating a conceptual model for an inter-professional approach to shared decision making: a mixed methods study. J Eval Clin Pract. 2011;17(4):554-64.

10. Yaghoubi M, Javadi M, Rakhsh F, Bahadori M. A study of determining factors affecting the performance of nurses based on the achieve model in selected hospital of Isfahan (Iran). J Educ Health Promot. 2013;2:49.

11. Gerdeman JL, Lux K, Jacko J. Using concept mapping to build clinical judgment skills. Nurse Educ Pract. 2013;13(1):11-7.

12. Glynn DM. Clinical judgment development using structured classroom reflective practice: a qualitative study. J Nurs Educ. 2012;51(3):134-9.

13. Traynor M, Boland M, Buus N. Professional autonomy in 21st century healthcare: nurses' accounts of clinical decision-making. Soc Sci Med. 2010;71(8):1506-12.

14. Skar R. The meaning of autonomy in nursing practice. J Clin Nurs. 2010;19(15-16):2226-34.

15. Gillet N, Colombat P, Michinov E, Pronost AM, Fouquereau E. Procedural justice, supervisor autonomy support, work satisfaction, organizational identification and job performance: the mediating role of need satisfaction and perceived organizational support. J Adv Nurs. 2013;69(11):2560-71.

16. Thompson C, Yang H, Crouch S. Clinical simulation fidelity and nurses' identification of critical event risk: a signal detection analysis. J Adv Nurs. 2012;68(11):2477-85.

17. Kantar L, Alexander R. Integration of clinical judgment in the nursing curriculum: challenges and perspectives. J Nurs Educ. 2012;51(8):444-53.

18. Jerak-Zuiderent S. Certain uncertainties: modes of patient safety in healthcare. Soc Stud Sci. 2012;42(5):732-52.

19. Victor-Chmil J, Larew C. Psychometric properties of the lasater clinical judgment rubric. Int J Nurs Educ Scholarsh. 2013;10.

20. Fero LJ, O'Donnell JM, Zullo TG, Dabbs AD, Kitutu J, Samosky JT, et al. Critical thinking skills in nursing students: comparison of simulation-based performance with metrics. $J$ Adv Nurs. 2010;66(10):2182-93.

21. Lockwood C. 179 pCochrane and nursing, moving forwards through new collaboration. Int J Nurs Pract. 2010;16(2):203-4.

22. Thompson C, Aitken L, Doran D, Dowding D. An agenda for clinical decision making and judgement in nursing research and education. Int J Nurs Stud. 2013;50(12):1720-6. 
23. Elliott N. 'Mutual intacting': a grounded theory study of clinical judgement practice issues. J Adv Nurs. 2010;66(12):2711-21.

24. Traynor M, Boland M, Buus N.Autonomy, evidence and intuition: nurses and decision-making. J Adv Nurs. 2010;66(7):1584-91.

25. Petrucci AM, Nouh T, Boutros M, Gagnon R, Meterissian SH. Assessing clinical judgment using the Script Concordance test: the importance of using specialty-specific experts to develop the scoring key. Am J Surg. 2013;205(2):137-40.

26. Gampel E. Does professional autonomy protect medical futility judgments? Bioethics. 2006;20(2):92-104

27. Wang Y, Chien WT, Twinn S. An exploratory study on baccalaureate-prepared nurses' perceptions regarding clinical decisionmaking in mainland China. J Clin Nurs. 2012;21(11-12):170 6-15.

28. Dickson GL, Flynn L. Nurses' clinical reasoning: processes and practices of medication safety. Qual Health Res. 2012;22(1):3-16.

29. Clarke JR. The role of decision skills and medical knowledge in the clinical jud gment of surgical residents. Surgery.1982;92(2):153-8.

30. Cranley LA, Doran DM, Tourangeau AE, Kushniruk A, Nagle L. Recognizing and responding to uncertainty: a grounded theory of nurses' uncertainty. Worldviews Evid Based Nurs. 2012;9(3):149-58.

31. Nakash O, Alegria M. Examination of the role of implicit clinical judgments during the mental health intake. Qual Health Res. 2013;23(5):645-54

32. Lasater K. Clinical judgment: the last frontier for evaluation. Nurse Educ Pract. 2011;11(2):86-92.

33. Lindsey PL, Jenkins S. Nursing students' clinical judgment regarding rapid response: the influence of a clinical simulation education intervention. Nurs Forum. 2013;48(1):61-70.
34. Foster KN, Lewis M, Marshall A, Lewis P. Educating Australian registered nurses in comprehensive health assessment: a pilot study. J Contin Educ Nurs. 2013;44(4):155-62.

35. Nielsen A. Concept-based learning activities using the clinical judgment model as a foundation for clinical learning. $J$ Nurs Educ. 2009;48(6):350-4.

36. Lasater K, Nielsen A. Reflective journaling for clinical judgment development and evaluation. J Nurse Edu. 2009;48(1):40-4.

37. Banning $M$. The think aloud approach as an educational tool to develop and assess clinical reasoning in undergraduate students. Nurse Educ Today. 2008;28(1):8-14.

38. Parker LE, Kirchner JE, Bonner LM, Fickel JJ, Ritchie MJ, Simons $\mathrm{CE}$, et al. Creating a quality-improvement dialogue: utiliz ing knowledge from frontline staff, managers, and experts to foster health care quality improvement. Qual Health Res 2009;19(2):229-42.

39. Bunkenborg G, Samuelson K, Akeson J, Poulsen I. Impact of professionalism in nursing on in-hospital bedside monitoring practice. J Adv Nurs. 2013;69(7):1466-77.

40. Goldsmith J, Wittenberg-Lyles E, Rodriguez D, Sanchez-Reilly S. Interdisciplinary geriatric and palliative care team narratives: collaboration practices and barriers. Qual Health Res. 2010;20(1):93-104.

41. Gholami Fesharaki M, Jamali MJ, Rahmati Najarkolaei F, Mohamadian M, Aghamiri Z. Validity and Reliability of the Najmiyeh Outpatients Satisfaction Questionnaire. Health Educ Health Promot. 2013;1(1):13-20. 